\title{
Short envelope pulse propagation in composite right- and left-handed transmission lines with regularly spaced Schottky varactors
}

\author{
Jun Ogasawara and Koichi Narahara ${ }^{a)}$ \\ Graduate School of Science and Engineering, Yamagata University, 4-3-16 Jonan, \\ Yonezawa, Yamagata 992-8510, Japan \\ a)narahara@yz.yamagata-u.ac.jp
}

\begin{abstract}
Composite right- and left-handed (CRLH) transmission lines with regularly spaced Schottky varactors are characterized for propagation of short envelope pulses. Due to strong dispersion, a short pulse cannot travel on a conventional CRLH line without distortion. This distortion is compensated for by the effects of Schottky varactors such that the line supports solitonic pulses, whose pulse width depends on the carrier frequency. It then becomes possible to find carrier frequencies at which the pulse width becomes minimal. This article describes the design criteria for propagating short envelope pulses in the lines.
\end{abstract}

Keywords: nonlinear Schrödinger solitons, CRLH lines, left-handed waves

Classification: Microwave and millimeter wave devices, circuits, and systems

\section{References}

[1] C. Caloz and T. Itoh, Electromagnetic metamaterials: transmission line theory and microwave applications, Wiley, New York, 2006.

[2] K. Narahara, T. Nakamichi, T. Suemitsu, T. Otsuji, and E. Sano, "Development of solitons in composite right- and left-handed transmission lines periodically loaded with Schottky varactors," J. Appl. Phys., vol. 102, pp. 024501-024504, 2007.

[3] T. Taniuti, "Reductive perturbation method and far fields of wave equations," Prog. Theor. Phys. Suppl., vol. 55, pp. 1-35, 1974.

[4] C. R. Paul, Analysis of multiconductor transmission lines, Wiley, New York, 1994. 


\section{Introduction}

It is well-known that a composite right- and left-handed (CRLH) transmission line defined as a lumped transmission line containing a series inductor, series capacitor, shunt inductor, and shunt capacitor in each section, exhibits lefthanded properties [1]. Because of dispersion, a broadband pulse cannot travel on the line without distortion. Here, we consider a modified CRLH line, whose shunt capacitor is replaced by a Schottky varactor, hereafter called a Schottky CRLH line. The nonlinearity introduced by the varactors can compensate for the dispersion of CRLH lines, resulting in an envelope soliton governed by the nonlinear Schrödinger equation. Lately, there have been discussions on the solitonic pulses in Schottky CRLH lines using second-order long-wavelength approximation [2]. However, the discrepancy between the approximated and exact dispersions is so large at short wavelengths that the existing formulation cannot describe the properties of short solitonic pulses. This article discusses the method of short-pulse propagation on Schottky CRLH lines, by improving the approximation accuracy together with the design criteria obtained analytically. Several numerical evaluation results validate this method.

\section{Short pulse in Schottky CRLH lines}

Figure 1 (a) shows a representation of a unit cell of a Schottky CRLH line. $L_{R}, C_{L}$, and $L_{L}$ represent the series inductor, series capacitor, and shunt inductor, respectively. $C_{R}$ represents the shunt Schottky varactor. $\Psi_{n}$ and $I_{n}$ indicate the line voltage and current at the $n$th cell. $V_{0}$ shows the terminal voltage of the Schottky varactor. The capacitance-voltage relationship of a Schottky varactor is generally given by

$$
C(V)=\frac{C_{0}}{\left(1-\frac{V}{V_{J}}\right)^{m}},
$$

where $C_{0}, V_{J}$, and $m$ are the optimizing parameters. Note that $V<0$ for reverse bias. Using these variables, the transmission equations of a Schottky CRLH line are given by

$$
\begin{aligned}
\left(L_{R} \frac{d^{2}}{d t^{2}}+\frac{1}{C_{L}}\right) I_{n} & =\frac{d}{d t}\left(\Psi_{n-1}-\Psi_{n}\right) \\
\left(C_{R}\left(\Psi_{n}-V_{0}\right) \frac{d^{2}}{d t^{2}}+\frac{1}{L_{L}}\right) V_{n} & =\frac{d}{d t}\left(I_{n}-I_{n+1}\right) \\
& -\left.\frac{d C_{R}(V)}{d V}\right|_{V=\Psi_{n}-V_{0}}\left(\frac{d \Psi_{n}}{d t}\right)^{2} .
\end{aligned}
$$

When the pulse spreads over many cells, the discrete spatial coordinate $n$ can be replaced by a continuous one $x$. For a broadband pulse, we series-expand $\Psi_{n \pm 1}$ around $\Psi_{n}$ up to the sixth order of the cell length. Applying this long-wavelength approximation to eqs. (2) and (3), we obtain the evolution equation of the line voltage:

$$
c_{R} c_{L} l_{R} l_{R} \frac{\partial^{4} \Psi}{\partial t^{4}}+4 c_{L} l_{R} l_{L} \frac{d c_{R}}{d V} \frac{\partial \Psi}{\partial t} \frac{\partial^{3} \Psi}{\partial t^{3}}+l_{L} \frac{d c_{R}}{d V}\left(\frac{\partial \Psi}{\partial t}\right)^{2}
$$




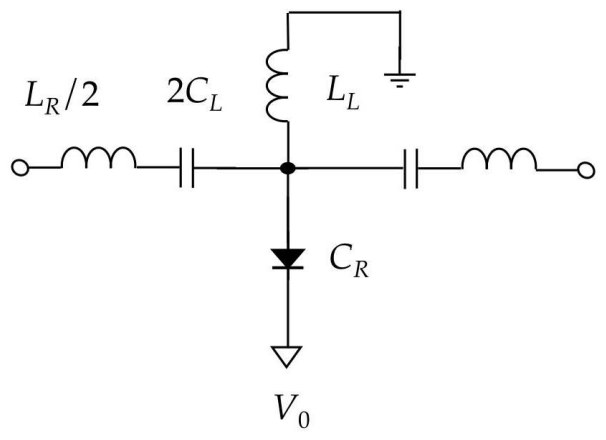

(a)

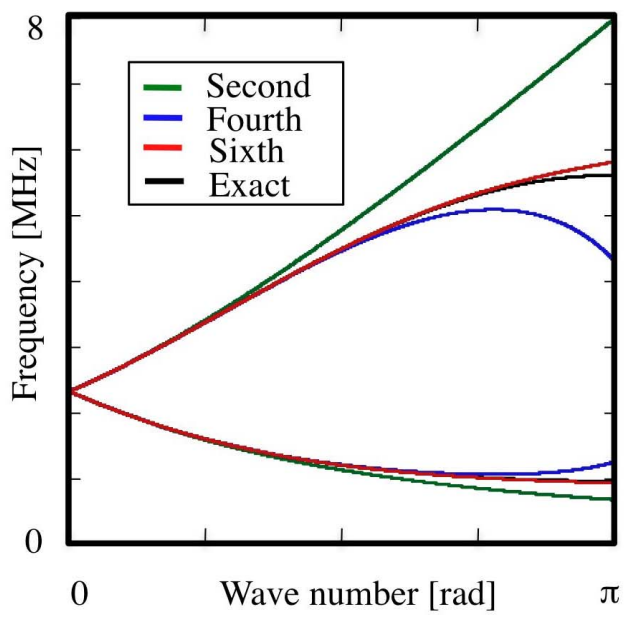

(b)

Fig. 1. Line properties: (a) equivalent representation of Schottky CRLH lines, and (b) dispersion of a CRLH line.

$$
\begin{aligned}
& +3 c_{L} l_{R} l_{L} \frac{d c_{R}}{d V}\left(\frac{\partial^{2} \Psi}{\partial t^{2}}\right)^{2}+6 c_{L} l_{R} l_{L} \frac{d^{2} c_{R}}{d V^{2}}\left(\frac{\partial \Psi}{\partial t}\right)^{2} \frac{\partial^{2} \Psi}{\partial t^{2}} \\
& +\quad c_{L} l_{R} l_{L} \frac{d^{3} c_{R}}{d V^{3}}\left(\frac{\partial \Psi}{\partial t}\right)^{4}+\left(c_{L} l_{R}+c_{R} l_{L}\right) \frac{\partial^{2} \Psi}{\partial t^{2}}+\Psi \\
& -\quad c_{L} l_{L} \frac{\partial^{4}}{\partial t^{2} \partial x^{2}}\left(\Psi+\frac{1}{12} \frac{\partial^{2} \Psi}{\partial x^{2}}+\frac{1}{360} \frac{\partial^{4} \Psi}{\partial x^{4}}\right)=0,
\end{aligned}
$$

where $l_{L, R}$ and $c_{L, R}$ are the line inductance and capacitance per unit length, respectively. The function $\Psi=\Psi(x, t)$ is the continuous counterpart of $\Psi_{n}$. The shunt capacitance at $V=0$, denote as $c_{R}^{(0)}$, determines the linear dispersion of the line. For brevity, we define $\omega_{\text {se }}$ and $\omega_{\text {sh }}$ as $1 / \sqrt{C_{L} L_{R}}$ and $1 / \sqrt{C_{R}^{(0)} L_{L}}$, respectively. Then, the line exhibits a left-handed (LH) property at frequencies lower than $\omega_{l} \equiv \min \left(\omega_{s e}, \omega_{s h}\right)$ and an ordinary righthanded $(\mathrm{RH})$ property at frequencies higher than $\omega_{u} \equiv \max \left(\omega_{s e}, \omega_{s h}\right)$. The LH branch is continuously connected with the RH one at $\omega=\omega_{u}=\omega_{l}$ in the balanced line. In contrast, a stop band, where all supporting modes become evanescent, appears between $\omega_{l}$ and $\omega_{u}$ in the unbalanced line. Therefore, we call the Schottky CRLH lines balanced when $C_{R}^{(0)} L_{L}=C_{L} L_{R}$ is satisfied, otherwise we call them unbalanced. The dispersion relationship $\omega=\omega_{R, L}(k)$ of the modes propagating on a linear CRLH line at the present approximation 
( $\omega_{R}$ is for the $\mathrm{RH}$ and $\omega_{L}$ for $\mathrm{LH}$ ) is expressed as

$$
\begin{aligned}
& \omega_{R}(k)=\sqrt{\omega_{x}^{2}(k)+\sqrt{\omega_{x}^{4}(k)-\frac{1}{c_{L} c_{R}^{(0)} l_{L} l_{R}}}} \\
& \omega_{L}(k)=\sqrt{\omega_{x}^{2}(k)-\sqrt{\omega_{x}^{4}(k)-\frac{1}{c_{L} c_{R}^{(0)} l_{L} l_{R}}}}
\end{aligned}
$$

where $\omega_{x}(k)$ is defined as

$$
\omega_{x}(k)=\sqrt{\frac{k^{6}}{720 c_{R}^{(0)} l_{R}}-\frac{k^{4}}{24 c_{R}^{(0)} l_{R}}+\frac{k^{2}}{2 c_{R}^{(0)} l_{R}}+\frac{1}{2 c_{R} l_{L}}+\frac{1}{2 c_{L} l_{R}} .}
$$

Figure 1 (b) shows the dispersion of a CRLH line. To obtain this, we set $L_{L}$, $L_{R}, C_{R}^{(0)}$ and $C_{L}$ to $100 \mu \mathrm{H}, 100 \mu \mathrm{H}, 47 \mathrm{pF}$ and $47 \mathrm{pF}$, respectively. Thus, the line is balanced. The black curve shows the exact dispersion, while the red curve shows the dispersion obtained by the present approximation. For reference, we indicate the dispersions obtained by the second- and fourthorder accuracy with green and blue curves, respectively. The latter two dispersions do not exactly match the one for short wavelengths, so they are inadequate for describing short pulses traveling on the line. Thus, we have to employ the sixth-order approximation. To quantify how the nonlinearity compensates for the dispersion, we extract the soliton equation from eq. (4) by reductive perturbation [3]. First, we prepare the spatial and temporal coordinates for the envelope and carrier waves. We use $x$ and $t$ as the spatial and temporal coordinates, respectively, to describe the carrier wave. For the envelope wave, $\xi \equiv \epsilon\left(x-V_{g}(k) t\right)$ and $\tau \equiv \epsilon^{2} t$ are the spatial and temporal coordinates, respectively, where $V_{g}(k)$ represents the group velocity of the line explicitly given by

$$
V_{g}(k)=\frac{k\left(k^{4}-20 k^{2}+120\right)}{240 C_{R} L_{R}} \frac{\omega(k)}{\omega_{x}^{2}(k)-\omega^{2}(k)},
$$

where $\omega(k)=\omega_{L}(k)$ for the LH branch and $\omega(k)=\omega_{R}(k)$ for the RH branch. Next, we expand the voltage variable as

$$
\Psi(x, t)=\sum_{m=1}^{\infty} \sum_{l=-\infty}^{\infty} \epsilon^{m} \psi_{l}^{(m)}(\xi, \tau) e^{i(\omega(k) t-k x)},
$$

for $\epsilon<<1$. Substituting this into eq. (4), the $\epsilon^{m}$ terms are collected to make them identically zero. By solving the resulting identities, we obtain the explicit solutions of $\psi_{l}^{(m)}$. Till the terms up to $O\left(\epsilon^{3}\right)$, it is found that $\psi_{-1}^{(1)}$ satisfies the following nonlinear Schrödinger (NS) equation,

$$
i \frac{\partial \psi_{-1}^{(1)}}{\partial \tau}+P(k) \frac{\partial^{2} \psi_{-1}^{(1)}}{\partial \xi^{2}}+Q(k)\left|\psi_{-1}^{(1)}\right|^{2} \psi_{-1}^{(1)}=0
$$

where the coefficients $P(k)$ and $Q(k)$ are given by

$$
P(k)=\frac{\left(k^{4}-12 k^{2}+24\right) \omega(k)}{96 C_{R} L_{R}\left(\omega_{x}^{2}(k)-\omega^{2}(k)\right)}+\frac{V_{g}(k)^{2}\left(5 \omega_{x}^{2}(k)-7 \omega^{2}(k)\right)}{2 \omega(k)\left(\omega_{x}^{2}(k)-\omega^{2}(k)\right)},
$$




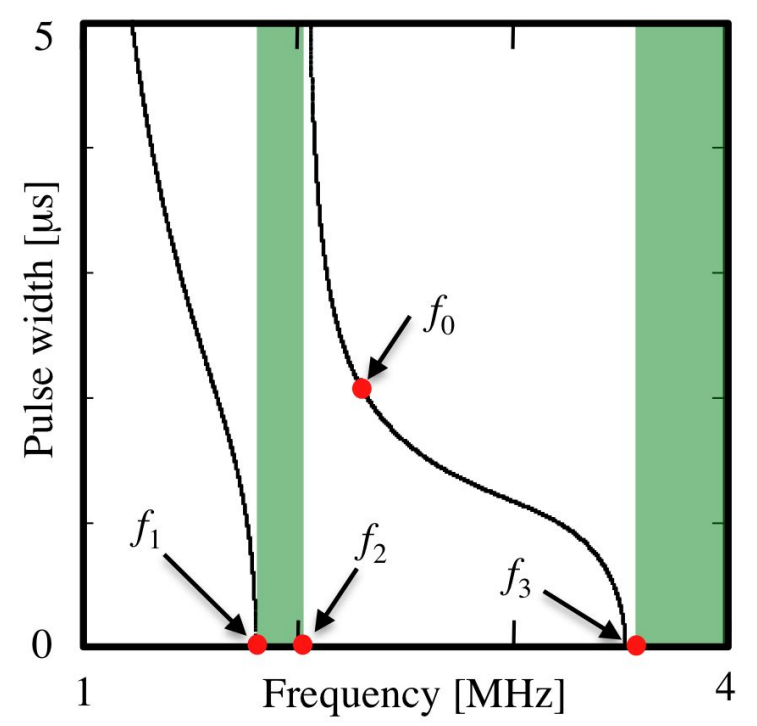

Fig. 2. Temporal widths of the solitonic pulses in Schottky CRLH lines.

$$
\begin{aligned}
Q(k) & =\frac{\omega(k)\left(-1+C_{L} L_{R} \omega^{2}(k)\right)}{8 C_{L} L_{R}\left(\omega_{x}^{2}(k)-\omega^{2}(k)\right)} \\
& \times \frac{M}{\left(V_{0}+V_{J}\right)^{2}}\left(1+\frac{1}{1-\frac{1}{\frac{1}{4 C_{R} L_{L} \omega^{2}(k)}+\frac{4 C_{L} k^{2}\left(45-15 k^{2}+2 k^{4}\right)}{45 C_{R}\left(-1+4 C_{L} L_{R} \omega^{2}(k)\right)}}} M\right)
\end{aligned}
$$

The light one-soliton solution of eq. (10) is given by

$$
\Psi(x, t)=A \operatorname{sech}\left(A \sqrt{\frac{Q(k)}{8 P(k)}}\left(x-V_{g}(k) t\right)\right) e^{i(\omega(k) t-k x)} .
$$

Note that $Q(k) / P(k)>0$ for the development of light solitons. The estimated pulse width is shown in Fig. 2. As a model of Schottky varactors, we set $C_{0}=64.77 \mathrm{pF}, V_{J}=3.561 \mathrm{~V}$ and $m=1.259$. $C_{L}$ is set to $47 \mathrm{pF} . L_{L}$ and $L_{R}$ are both set to $100 \mu \mathrm{H}$. Moreover, $V_{0}$ is set to $+1 \mathrm{~V}$, and the pulse amplitude $A$ is set to $1 \mathrm{~V}$. The frequencies $f_{0}, f_{1}, f_{2}$, and $f_{3}$ are 2.32, 1.82, 2.04, and $3.55 \mathrm{MHz}$, respectively. The line exhibits the $\mathrm{RH}$ and $\mathrm{LH}$ properties at frequencies above and below $f_{0}$, respectively. The hatched regions in Fig. 2 bounded by $f_{1,2}$ or $f_{3}$, represent the frequencies at which the light solitons cannot be allowed to develop. We can see that the pulse width becomes very short at frequencies either around $f_{1}$ or around $f_{3}$. These observations are useful in designing the carrier frequency and for signal application.

\section{Numerical evaluation}

We numerically solve eqs. (2) and (3) using the standard finite-difference time-domain method [4] for a Schottky CRLH line. The total cell size is 2000. The parameters are the same as those used to obtain Fig. 2. The input pulse has the form of eq. (14) with an amplitude $A$ of 0.3 . The carrier frequency is $1.7 \mathrm{MHz}$. Note that the line exhibits the LH property at this frequency. We compared the pulse propagation in a nonlinear Schottky CRLH line with 


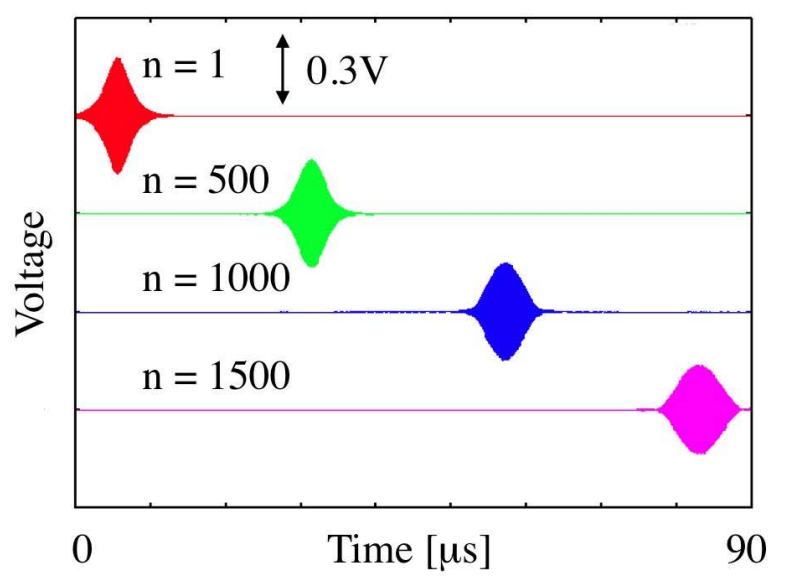

(a)

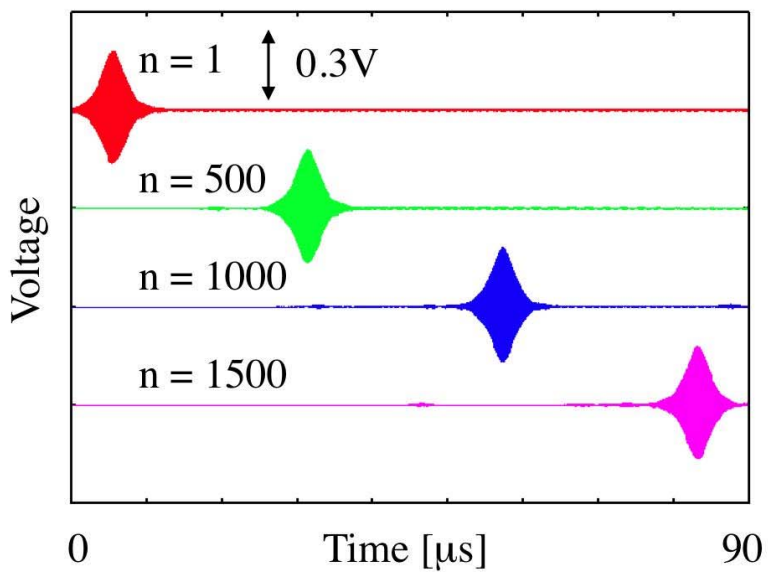

(b)

Fig. 3. Numerical evaluations. The waveforms on (a) the linear and (b) nonlinear CRLH lines.

that in a linear CRLH line. For the linear line, we employ a $47 \mathrm{pF}$ capacitor in place of the Schottky varactor.

Figure 3 (a) shows the numerically obtained waveforms monitored for the linear line at cells $n=1,500,1000$, and 1500. Due to the dispersion, the waveform gradually becomes distorted during propagation. Figure 3 (b) shows the waveforms for the Schottky CRLH line. In contrast to the linear line, the pulse travels without distortions in every cell. By the calculations using the carrier frequency of $3.4 \mathrm{MHz}$, it was found that the line supported an $\mathrm{RH}$ solitonic pulse. Moreover, the calculations at $2.0 \mathrm{MHz}$ led to the observation that both the dispersion and nonlinearity stretched out the input pulse, resulting in a greatly distorted waveform.

In conclusion, we described the propagation of short envelope pulses in Schottky CRLH lines. The Schottky varactors employed at $C_{R}$ make the CRLH line a good platform for developing short envelope pulses and we expect to see them in a variety of broadband applications. 